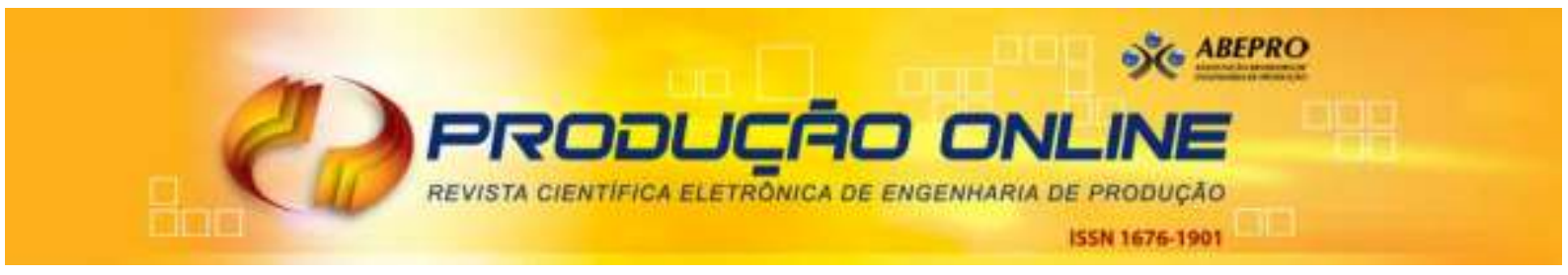

\title{
DIAGNÓSTICO DO PROCESSO DE BERLINER, COMPORTAMENTO ELEMENTAR E MODO DE ERRO CAUSADORES DE INTERRUPÇÕES DE ENERGIA ELÉTRICA
}

\section{DIAGNOSTIC OF BERLINER PROCESS, ELEMENTARY BEHAVIOR AND ERROR MODE CAUSING ELECTRICITY INTERRUPTION}

\author{
Vanessa Nóbrega da Silva* E-mail: vanessanobres7@gmail.com \\ Márcio José das Chagas Moura* E-mail: maciocmoura@gmail.com \\ Suelyn Fabiana Aciole Morais* E-mail: suelyn.morais@hotmail.com \\ *Universidade Federal de Pernambuco (UFPE), Recife, PE
}

\begin{abstract}
Resumo: O desempenho humano tem sido estudado em face às limitações impostas ao ambiente e que refletem no desempenho do colaborador. Para que os erros humanos sejam minimizados é necessário que sejam analisados e propostas medidas mitigatórias. O objetivo do estudo é adicionar o tipo de erro "lapso" a estrutura proposta por Vilela (2013). Posteriormente, é realizada uma análise de agrupamentos para observar quais modos de falha, comportamento elementar e processo de Berliner que causaram mais interrupções de energia elétrica entre os anos de 1998 e 2009. Para tanto, a metodologia foi baseada em uma análise de agrupamentos a qual auxiliou na divisão dos erros humanos por grupos de características semelhantes, fornecendo assim uma identificação mais realística do comportamento humano que ocasionou o erro. Por conseguinte, essa pesquisa fornece suporte para uma proposição de soluções que possam contribuir para a redução dos erros humanos. O resultado da análise de agrupamento identificou os grupos 3 e 4 como os maiores ocasionadores de interrupções de energia elétrica. Também foi realizada a mensuração dos custos envolvidos na interrupção de energia elétrica, evidenciado o alto valor do faturamento perdido pela empresa no período estudado.
\end{abstract}

Palavras-Chave: Confiabilidade Humana. Erro Humano. Taxonomia. Análise de Agrupamentos. Mensuração dos Custos. Setor Elétrico.

Abstract: Human performance has been studied in face of limitations imposed on the environment which reflect the performance of the employee. So that human errors are minimized it is necessary to analyze them, proposing mitigation actions. The aim of this research is adding error type "lapse" to the structure proposed by Vilela (2013). Subsequently it is carried out an analysis of groupings to observe which failure modes, basic behavior and Berliner process caused more electricity disruption between 1998 and 2009. To this end, the methodology was based on a cluster analysis which assisted in the division of human errors by groups of similar characteristics, thus providing a more realistic identification of human behavior which caused the error. Therefore this research provides support for a proposition of solutions that may contribute to the reduction of human errors. The result of cluster analysis identified the groups 3 and 4 as major sources of electric power outages. The measurement of costs involved in electric power interruption was also performed, which evidences the high loss of company's revenue in the studied period.

Keywords: Human Reliability. Human Error. Taxonomy. Cluster Analysis. Measurement Costs. Electrical Sector. 


\section{INTRODUÇÃO}

A confiabilidade humana possui um papel fundamental no que tange ao desenvolvimento das atividades em uma organização tendo sua definição relacionada ao entendimento das restrições e limitações que atuam sobre o desempenho de um indivíduo e que podem levá-lo ao erro (SWAIN \& GUTTMAN, 1983).

Dentro dessa perspectiva é importante considerar que os eventos que decorrem da ausência de confiabilidade humana e que pré-dispõem ao erro, devem ser observados sob a ótica da multidisciplinaridade. À luz de Siciliano e Ferraz (2010) uma atividade deve ser executada de maneira que os eventos indesejáveis são prevenidos e em seguida são sugeridos formas de minimização dos erros humanos. Esses erros são decorrentes das ações errôneas e produzem um resultado previsto e/ou reproduz uma consequência não desejada (FILGUEIRAS, 1996).

Carvalho et al., (2002) garantem que a análise do erro humano passa por fases de investigação e reconstituição do erro, observando o porquê, suas causas dentre outras informações que fornecerão embasamento sobre o erro cometido, para que posteriormente, possa ser realizada uma avaliação e correção do mesmo com seleção e aplicação das ferramentas mais adequadas para que os riscos aos colaboradores sejam identificados, quantificados e se possível, reduzidos.

Os erros humanos também são responsáveis por grande parte dos apagões ocorridos no setor elétrico nos últimos anos e sobre estes incidem custos associados por perda de faturamento bem como perda da imagem da empresa frente ao mercado consumidor. Diante dessa abordagem, a coleta, organização e análise de dados sobre o erro humano pode oferecer benefícios para os estudos sobre confiabilidade humana dada a constatação da existência dos eventos citados.

Nesse sentido, estudos realizados por Vilela (2013) sobre os erros humanos no setor elétrico entre os anos de 1998 a 2009, abordaram os tipos de erro "engano" e "deslize" como os responsáveis pelos maiores índices de interrupção de energia. Sobre este estudo incide a ausência do tipo de erro "lapso" o qual quando considerado fornece mais embasamento sobre os tipos de erros humanos envolvidos quando na motivação da interrupção de energia. De posse dessa informação foi realizada uma re-leitura com o acréscimo do "lapso" ao estudo proposto por Vilela e realizado posteriormente uma re-leitura desses erros para em seguida, realizar uma análise multivariada, a qual forneceu uma quantidade de grupos responsáveis pelos maiores índices de interrupção de energia elétrica bem como os seus principais elementos causadores. Após este exame, foi realizada uma estimativa dos custos incorridos nas interrupções ao longo dos anos já referidos com o objetivo de ilustrar o valor da perda de faturamento pela empresa devido aos blecautes ao longo dos anos estudados.

Logo, o objetivo da pesquisa, é identificar os modos de erro responsáveis pela interrupção de energia elétrica, através do diagnóstico do processo de Berliner 
(1964), o qual pontua que o erro humano é composto pelos processos cognitivos (relacionados à resolução de problemas e tomadas de decisão), perceptivos (associado à procura e recebimento de informações), motor (pautado a processos de movimentação de equipamentos e/ou maquinários, podendo ser simples e discretos, complexos e contínuos) e de comunicação (são os feedbacks necessários ao melhor andamento das atividades).

\section{FUNDAMENTAÇÃO TEÓRICA}

\subsection{Confiabilidade Humana}

A maneira como um sistema executa suas funções diante das condições físicas, emocionais e ambientais pré-existentes são fatores que contribuem para a construção do arquétipo de desempenho humano, utilizando para isto uma Análise de Confiabilidade Humana ( $\mathrm{ACH}$ ), a qual "estuda a execução das ações humanas em um determinado sistema, considerando os fatores que influenciam no seu desempenho, os quais comumente evidenciam a incompatibilidade existente entre as limitações humanas e as condições impostas para o trabalho" (DROGUETT \& MENÊZES, 2007).

Nesse contexto, a detecção de fatores humanos relacionados à execução de uma atividade é substancial para que o contexto de uma análise de confiabilidade seja devidamente conhecido, analisado e fornecido(s) meio(s) de se atenuar um possível erro. Calixto (2011) afirma que a detecção dos fatores humanos na abordagem da confiabilidade humana está alicerçada em quatro segmentos: psicológicos, fisiológicos, tecnológicos e sociais. De maneira geral, não avaliar esses fatores psicológicos e organizacionais, resulta em um entendimento sobre confiabilidade humana inadequado, uma vez que as análises realizadas sobre a dependência são irreais e não representam adequadamente 0 procedimento humano.

É importante considerar que o cenário que pré-dispõe ao erro, não é isolado dos eventos dos erros humanos e, quando estes dois fatores (ambiente e humano) são associados, podem interferir no desempenho físico, emocional e cognitivo do indivíduo, acarretando assim o erro e, por conseguinte, fazendo com que a organização tenha suas atividades, qualidades e credibilidades de seus ativos comprometidas diante dos consumidores e concorrentes. Ribeiro (2012) argumenta que o erro humano não deveria ter conotação de culpa e sim ser a base para que os gestores verifiquem as situações de trabalho e as causas primordiais que induzem ao erro.

\subsubsection{Erro Humano}

Sanders \& McCormick (1993) afirmam que o erro é toda ação, engano, falso juízo que leva o elemento humano a cometer um engano, e que pode reduzir o 
desempenho e segurança do sistema. Swain e Guttmann (1983) contextualizam erro humano como sendo a probabilidade da confiabilidade humana sob dois aspectos: execução correta de uma atividade/tarefa exigida pelo sistema dentro de um espaço de tempo solicitado; não realização de atividade que possa ocasionar a destituição do sistema.

As consequências que decorrem dos erros humanos são por natureza, dúbias e podem ser consideradas sob aspectos como: deslizes (não constituem indicativos de falta de conhecimento das regras vigentes nem pressupõem uma ação intencional de violação, mas são sempre decorrentes de condições extemporâneas como estresse ou desatenção); enganos -mistake (manifesta-se quando não há harmonização entre a meta selecionada e a determinação do plano, ou seja, mesmo que as ações transcorram como planejadas, o plano pode não estar adequado para que a tarefa seja executada com êxito) e lapsos - lapse (está relacionado à falta de memória/esquecimento de uma tarefa ou etapa dela por parte do colaborador) (REASON, 1990; BEGOSSO, 2005; FILHO et al., (2009).

Correa e Júnior (2007) asseguram que a diferença básica entre os deslizes, lapsos e enganos é que os primeiros correspondem à acepção habitual de erro e os enganos tratam de questões como conhecimento, objetivos, raciocínio do operador. Em contrapartida, Santos (2012) afirma que os deslizes e os lapsos têm lugar quando as ações se afastam da intenção devido à deficiência na execução. Por outro lado, os enganos surgem quando as ações se desenvolvem segundo um plano inadequado para atingir o objetivo desejado.

Nesse sentido, os erros podem decorrer tanto da decodificação errônea de uma atividade, como da cognição do trabalhador, onde esta envolve além do estado emocional do trabalhador, a maneira como o homem entende a atividade e seus desdobramentos. Já sob a ótica de Rasmussem (1986), o erro humano pode ser percebido sob três aspectos:

1) Nível de regras (Rule Based): o erro decorre da seleção e/ou aplicação de conceitos inadequados a solução do problema.

2) Nível de conhecimento (Knowledge Based): quando em novas situações a melhor maneira de solucionar o problema é realizada de atitude consciente.

3) Nível de habilidade (Skill Based): por haver um conjunto de tarefas que cotidianamente são executadas, o nível de resposta é automático.

À luz de Berliner (1964) o erro humano pode ser visto de acordo com o comportamento elementar por ele desenvolvido e é dividido em 32 verbos segmentados em 4 grupos (processos cognitivos, perceptivos, motor e de comunicação) que definem o tipo de atividade que o colaborador estava realizando quando no momento do erro. O Quadro 2.1 ilustra a estrutura da taxonomia proposta por Berliner. 
Quadro 2.9 - Modelo proposto por Berliner

\begin{tabular}{|c|c|c|c|}
\hline $\begin{array}{l}\text { PROCESSO } \\
\text { COGNITIVO }\end{array}$ & $\begin{array}{c}\text { PROCESSO } \\
\text { PERCEPTIVO }\end{array}$ & PROCESSO MOTOR & $\begin{array}{l}\text { PROCESSO DE } \\
\text { COMUNICAÇÃO }\end{array}$ \\
\hline Calcular & Inspecionar & Mover & Responder \\
\hline Escolher & Observar & Segurar & Informar \\
\hline Dividir & Ler & Puxar/impulsionar & Pedir \\
\hline Comparar & Monitorar & Dar & Registrar \\
\hline Interpolar & Explorar & Remover & Ordenar \\
\hline Verificar & Detectar & Descartar & \multirow[t]{6}{*}{ Receber } \\
\hline \multirow[t]{5}{*}{ Lembrar } & Identificar & Devolver & \\
\hline & \multirow[t]{4}{*}{ Localizar } & Posicionar & \\
\hline & & Ajustar & \\
\hline & & Digitar & \\
\hline & & Instalar & \\
\hline
\end{tabular}

Fonte: Begosso (2005)

De posse dos verbos que foram ocasionadores do erro, é indicado o tipo de processo onde teve início à possível falha, se cognitivo se perceptivo, se motor ou de comunicação.

Nesse sentido, a junção dos modelos de Berliner (1964), Rasmussem (1983), Reason (1990) e Vilela (2013) são fundamentais para a adaptação de uma taxonomia que auxilie na identificação do erro humano. Essa taxonomia está baseada em cinco palavras para classificação do erro humano:

i. O processo de Berliner: composto pelos processos cognitivos (relacionados à resolução de problemas e tomadas de decisão), perceptivos (associado à procura e recebimento de informações), motor (pautado a processos de movimentação de equipamentos e/ou maquinários, podendo ser simples e discretos, complexos e contínuos) e de comunicação (são os feedbacks necessários ao melhor andamento das atividades);

ii. Nível de erro: são os níveis de regra, níveis de conhecimento e os níveis de habilidade. Os erros nos níveis de habilidade apresentam comportamentos automáticos, misturados a comportamentos conscientes, segundo BEGOSSO (2005).

iii. Comportamento elementar: composto por 32 verbos que, divididos entre os quatro processos de Berliner, definem o tipo de atividade que estava sendo realizada quando no momento do erro;

iv. Modo de erro: cada modo de erro está associado a 28 maneiras de avaliar como o erro aconteceu, se por falta de habilidade nas atividades rotineiras (if skill), se por falta de conhecimento das regras dispostas (if rule) ou se por falta de experiência para exercer a atividade (if knowledge);

v. Tipo de erro: são os deslizes (slips), enganos (mistake) e lapsos. 
Menêzes (2005) reportando a idéia de como o erro humano tem sido abordado, afirma que "o erro humano poderá ter diferentes causas, sendo necessárias diferentes medidas para prevení-lo" e considera que é necessário conhecê-los em toda sua extensão para então ser possível a proposição de métodos de $\mathrm{ACH}$ para uma posterior identificação e sistematização dos métodos que se farão úteis para quantificação do erro humano.

Não obstante, a confiabilidade humana possui seu significado além do termo "erro humano" o que significa afirmar que quando o resultado desejado é alcançado dentro de todas as condições estabelecidas para o processo e sem intercorrências, tem-se que a atividade foi executada de maneira eficiente e eficaz.

\subsection{O Setor Elétrico}

Diante do crescimento gradual do consumo de energia hidrelétrica, sendo o Brasil um dos líderes mundiais na produção deste tipo de energia e possuir cerca de $12 \%$ de água doce superficial com um alto potencial hidrelétrico estimado em cerca de 260 GW, dos quais 40,5\% estão localizados na Bacia Hidrográfica do Amazonas, o planejamento inadequado dessa energia atrelado a energia desperdiçada, aos blecautes ocorridos bem como as outras maneiras disponíveis de geração de energia que não são aproveitadas (a exemplo da energia eólica, solar, biomassa dentre outros) impedem que o País desenvolva todo o seu potencial energético (PORTAL BRASIL, 2010; BBC BRASIL, 2012; FOLHA DE SÃO PAULO, 2012).

O panorama exposto faz com que a sociedade torne-se mais exigente quanto à prestação de serviço desse setor, já que tem havido uma maior consciência sobre a necessidade da sustentabilidade no fornecimento de energia elétrica, bem como uma maior confiabilidade e qualidade nessa distribuição. Por causa dessas questões é que a realização de estudos no que tange à geração, transmissão e distribuição de energia tem se tornado importante já que os acidentes/incidentes ocorridos no setor advém em sua maior parte dos erros humanos. A falta de conhecimento, o não cumprimento de regras, informações necessárias para uma execução eficaz da atividade, tende a ser um fator que influencia diretamente na ocorrência destes erros e comprometem além da sua saúde física e emocional do indivíduo, toda a organização.

Nesse contexto, Righi, et al., (2010), declaram que as informações advindas dos erros gerados são primordiais na análise dos acidentes, visto que os elementos responsáveis pelo erro fornecem a base para a gestão dos deslizes, enganos, lapsos, falhas e seus desdobramentos, enfim, todas as intercorrências que possam ser relacionadas ao erro humano. Os autores certificam ainda que a questão dos erros não englobam apenas os trabalhadores envolvidos, mas abrange também o modo como a organização desenvolve suas atividades, seu ambiente de trabalho, as responsabilidades que ela atribui a seus funcionários dentre outros aspectos, são fatores que quando considerados dão subsídios para que o erro humano seja visto mais holisticamente. 


\subsection{Análise de Agrupamentos}

A Análise de Agrupamentos segundo é um procedimento explicativo que auxilia no entendimento de questões complexas das relações multivariadas e que ajuda na melhor visualização do universo amostral para o desenvolvimento de uma metodologia que classifique e represente os dados de maneira mais eficiente (LINDEN, 2009). Bussab et al., (1990) afirmam que "análise de agrupamentos engloba uma variedade de técnicas e algoritmos cujo objetivo é encontrar e separar objetos em grupos similares."

A importância da análise de agrupamentos está fundamentada no que concerne à segmentação e, posteriormente, à formação de grupos dos dados disponíveis para análise, de modo que as relações entre as variáveis estudadas sejam capazes de indicar a homogeneidade dentro dos grupos e a heterogeneidade entre os eles, para que, dessa maneira, seja possível a proposição de classificações.

A estrutura básica da aplicação de técnicas de análise de agrupamentos pode ser decomposta nas seguintes etapas: formulação do problema, obtenção e tratamento dos dados, escolha do critério de similaridade ou dissimilaridade, classificação das técnicas de agrupamento (método hierárquico ou não-hierárquico), apresentação dos resultados e por fim, avaliação e interpretação dos resultados.

É importante considerar que as variáveis escolhidas apresentem semelhanças entre os elementos de maneira que possa haver a padronização dos dados para um posterior agrupamento, e que as variáveis consideradas não sejam medidas na mesma unidade para que elas não contribuam igualmente para a similaridade entre os indivíduos ou ainda que tenham alguma influência arbitrária nos grupos formados (MEYER, 2002).

\subsubsection{Medidas de Similaridade e Dissimilaridade}

Para as medidas de similaridade, o método de agrupamento escolhido para a análise de dados de uma pesquisa e um coeficiente de correlação que quantifique a semelhança entre dois objetos são itens de fundamental importância na análise de agrupamento (MARTINS, 2008; VICINI \& SOUZA, 2005).

$\mathrm{O}$ coeficiente de correlação está relacionado à medida do grau de associação entre valores pela representação de pontos num sistema de coordenadas e suas respectivas posições em relação a uma linha reta. Quanto maior for este coeficiente, mais parecidos são os elementos da amostra. 


\subsubsection{Classificação das Técnicas de Agrupamento}

\subsubsection{Métodos Hierárquicos}

Doni (2004) afirma que o método hierárquico consiste em uma série de sucessivos agrupamentos ou sucessivas divisões de elementos, onde os elementos são agregados ou desagregados. São os métodos que dão forma a análise de agrupamentos e estão disponíveis em diversos softwares estatísticos. Compete ao pesquisador conhecer e saber qual método mais se adéqua a análise que se pretende realizar. Segundo Anderberg (1973), os métodos hierárquicos são:

1. Método simples: baseiam-se na categorização acumulada dos dados onde "os agrupamentos entre objetos ou entre grupos são feitos por ligações simples entre pares de objetos, ou seja, a distância entre os grupos é definida como sendo aquela entre os objetos mais parecidos entre esses grupos."

2. Método completo: segundo Frei (2006) é caracterizado pela similaridade entre os elementos de dois grupos que menos se parecem, ou seja, observa-se a distância máxima entre o primeiro grupo e os objetos distantes, aproximando os dois elementos mais parecidos e de menor distância.

3. Método dos centróides: está relacionado à distância do centro de massa entre dois grupos com o objetivo de unir os grupos que possuem a menor distância entre si, através dos pontos definidos pelas médias das variáveis caracterizadoras dos indivíduos de cada grupo, ou seja, o método do centróide calcula a distância entre dois grupos como a diferença entre as suas médias, para todas as variáveis (ALBUQUERQUE, 2005).

4. Método da soma de erros quadráticos ou variância (método Ward): está alicerçado, na individualidade de cada elemento formar um único grupo. Considerando a primeira reunião de elementos em um novo agrupamento, Albuquerque (2005) afirma que a soma dos desvios dos pontos representativos de seus elementos, em relação à média do agrupamento, é calculada e dá uma indicação de homogeneidade do agrupamento formado.

\subsubsection{Métodos Não Hierárquicos}

Ocorre também que os métodos não hierárquicos podem ser considerados como métodos que auxiliam nos agrupamentos, são eles:

1. Os métodos das k-médias: é usado quando se tem muitos elementos a serem analisados e cada elemento da amostra é recolocado a menor distância cujo vetor de médias é o mais próximo do vetor de valores 
observados para o respectivo elemento. Inicialmente escolhe-se o que será homogêneo dentro de cada grupo e o que será heterogêneo entre os grupos.

2. Os métodos dos medóides: está relacionado à característica que cada elemento é representado como sendo o centro do seu próprio grupo onde o medóide de cada grupo está relacionado ao estabelecimento dos kgrupos para designar os $n$ elementos que pertencem a um conjunto $X$, selecionando de maneira aleatória os k-objetos medóides iniciais, ou seja, utiliza-se o valor médio dos elementos em um grupo como um ponto de referência, chamado de medóide (DONI, 2004). Posteriormente, cada um dos $n-k$ objetos remanescentes é alocado ao grupo cujo medóide está mais próximo, realizando inúmeras tentativas de troca de medóides por não medóides e reavaliar qualidade dos novos agrupamentos resultantes (BRITO et al., 2011).

\subsubsection{Diferenças entre os Métodos Hierárquicos e Não Hierárquicos}

Matos (2007) corrobora que os métodos hierárquicos podem ser classificados em aglomerativos e divisivos. O método aglomerativo inicia-se com os grupos separados e eles vão sendo unidos em grupos cada vez maiores até que formem um único grupo. Já o método divisivo na ótica de Vicini \& Souza (2005) tem início com um único grupo e, posteriormente, é segmentado em grupos menores e na mesma quantidade de grupos existentes (respeitando a semelhança dos elementos dentro dos grupos).

\subsubsection{Técnicas de validação dos agrupamentos}

O julgamento de uma análise de agrupamento no que tange à qualidade dos dados estudados é realizado de maneira qualitativa através de gráficos que validam ou não, os grupos formados (MARTINS, 2008). Dentre estas técnicas estão o gráfico de silhueta e o gráfico de perfil. $O$ gráfico de silhueta ilustra os elementos, que classificados na análise de agrupamentos, podem estar bem alocados $(x>1)$ ou mal alocados $(x<0)$, de acordo com a distância estabelecida e os critérios de proximidade. Martins (2008) diz que o gráfico de perfil objetiva averiguar a qualidade dos agrupamentos e fazer com que a interpretação seja mais rica em detalhes, em que no eixo das abscissas ( $x$ ) estão às variáveis estudadas e no eixo das ordenadas (y) estão às escalas de medidas, com cada média é representada por um ponto nos eixos cartesianos e, com a união dos pontos, observam-se os perfis dos grupos.

\subsubsection{Correlação Cofenética}

A correlação cofenética segundo Barroso \& Artes (2003) está baseada nas comparações entre as distâncias calculadas a partir do agrupamento e aquelas que 
de fato foram observadas e são usadas com maior freqüência nos métodos de agrupamentos hierárquicos. Albuquerque (2005) assegura que quanto mais próximo a um for 0 valor da correlação cofenética, menor será a distorção provocada pelo agrupamento dos indivíduos com os métodos, ou seja, uma correlação cofenética em torno de 0,8 já pode ser considerada um bom ajuste.

\subsubsection{Soma dos Quadrados Dentro dos Grupos da Partição (SQDP)}

A soma dos quadrados dos grupos da partição (SQDP) parte do princípio da escolha do número final de grupos " $G$ " para saber com quantos grupos a análise será iniciada, ou onde o algoritmo deve ser interrompido. A Equação 2.1 é utilizada para construir o gráfico que servirá para definir o número inicial $\mathrm{G}$ de grupos.

$G=\frac{S Q D P(k)-S Q D P(k+1)}{S Q D P(k+1)}$

Quanto menor o valor de $\mathrm{G}$ menor é a vantagem de se trabalhar com um número maior de grupos.

\subsection{Custos de Interrupção de Energia Elétrica}

A qualidade nos serviços de energia elétrica está fortemente relacionada com o âmbito econômico dado os impactos financeiros de uma sobre a outra. Nesse sentido, verificam-se que os problemas relacionados à qualidade de energia elétrica possuem custos de interrupção que causam prejuízos às empresas concessionárias de energia e implicam nos valores das tarifas a serem cobradas pelas distribuidoras. Observa-se que esses custos de interrupção de energia são diferentes para cada tipo de classe consumidora, quais sejam: industrial, residencial, comercial, poder público dentre outros e, a determinação destes custos é de difícil realização pelas inúmeras variáveis subjetivas envolvidas (SHIGA et al., 2006).

Segundo Cruz (2007), as concessionárias de energia elétrica pontuam os custos de interrupção de energia sob seis aspectos:

i. Perdas de faturamento associadas à energia não suprida;

ii. Custos de reparos e manutenção no sistema para restabelecimento do serviço;

iii. Multas impostas pela ANEEL quando a empresa não cumpre as metas de continuidade;

iv. Ressarcimento de prejuízos causados a consumidores;

v. Gastos com campanhas publicitárias para melhoria de imagem junto a seus clientes;

vi. Redução de lucros associados à energia gerada pelos próprios consumidores. O referido autor pontua ainda que a perda de faturamento associada à energia não suprida pode ser calculada através do produto entre a tarifa da energia $(\mathrm{R} \$ / K w h)$ e o montante $(\mathrm{kWh})$ não fornecido, sendo que este valor é pouco significativo em relação às perdas sofridas pelos consumidores. É importante 
considerar que o custo de interrupção varia de acordo com o horário de interrupção, período do ano, mês a mês e é diferente para cada tipo de consumidor (SHIGA, 2007).

\section{METODOLOGIA}

Os dados foram obtidos através do levantamento sobre erros humanos feitos por Vilela (2013) os quais são descritos 131 erros que causaram apagões, decorridos ao longo de 11 anos. Posteriormente ao estudo da tese deste autor, foi realizada a ampliação da taxonomia através da inclusão do tipo de erro "lapso" (já que na base da dados este tipo de erro não era considerado) dada à necessidade de se conhecer quais erros eram decorrentes da falta de memória ou esquecimento do indivíduo que estava realizando a atividade, deixando assim o modelo mais completo, pois dessa maneira se dispõe de um modelo completo para análise do erro humano. Em seguida, foi realizado um re-ordenamento dos erros adequando-os ao "lapso" identificando quais eram os fatores causadores das interrupções de energia.

\subsubsection{Tratamento dos dados}

Quanto à padronização quantitativa, os grupos foram formados considerando as similaridades e dissimilaridades onde quanto mais semelhantes fossem, menor a distância euclidiana entre eles e, quanto menos semelhantes maior seria esta mesma distância. Logo, a padronização foi realizada de acordo com as similaridades ou dissimilaridades entre os agrupamentos. No que tange à padronização qualitativa, como se dispõe de dados nominais, usou-se a transformação dos dados em variáveis binárias para poder medir o grau de similaridade entre os elementos de um grupo.

\subsubsection{Escolha de critérios de similaridade}

Foram realizados testes com os métodos hierárquicos: simples, completo, do centróide e de ward e os métodos não hierárquicos das k-médias e dos medóides, para $K=2,3$, 4 e 5 grupos, obtendo para cada $K$ de cada método, um gráfico de silhueta e um gráfico de perfil. A escolha do método foi realizada baseada nos métodos de validação da análise de agrupamentos são eles: gráficos de silhueta, coeficiente de correlação cofenético, gráfico de perfil e índice G.

\subsubsection{Adoção e execução dos métodos hierárquicos e não hierárquicos}

Após escolha dos critérios de similaridade e testá-los para os agrupamentos de $K=2,3,4$ e 5, utilizou-se do coeficiente cofenético e do cálculo do 
índice $\mathrm{G}$ dos grupos até se obter o melhor agrupamento para se trabalhar na análise de dados.

\subsubsection{Apresentação dos resultados}

Com os resultados dos métodos aplicados e a alocação de cada um dos 131 elementos em cada grupo designado pela análise de agrupamentos, os resultados foram apreciados à luz dos agrupamentos de elementos que causaram maior interrupção de energia elétrica.

\subsubsection{Avaliação e interpretação dos resultados}

Os resultados foram avaliados de acordo com as variáveis taxonômicas propostas como: processo de Berliner, nível de erro, comportamento elementar, modo de erro e tipo de erro e interpretados sob a ótica da quantidade de energia que deixou de ser fornecida (Mwh). Inicialmente realizou-se um diagnóstico do processo de Berliner, comportamento elementar e modo de erro que são responsáveis pelas maiores interrupções de energia elétrica. A segunda etapa foi realizada através da ampliação da base de dados. A terceira etapa deu-se com o tratamento dos dados considerando as similaridades e dissimilaridades entre os grupos e em seguida, transformou os dados qualitativos em variáveis binárias. A quarta etapa deu-se com a escolha dos critérios de similaridade e dissimilaridade. Na quinta etapa foi realizada a adoção e execução dos métodos hierárquicos e não hierárquicos. $\mathrm{Na}$ sexta etapa, apresentou-se os resultados à luz dos grupos que fornecem uma maior quantidade de energia elétrica interrompida e por fim, os resultados foram avaliados de acordo com as variáveis taxonômicas propostas e interpretados sob a ótica da quantidade de energia elétrica que deixou de ser fornecida (Mwh)

\subsection{Estimação dos custos de interrupção de energia elétrica}

Os custos de interrupção de energia elétrica foram estimados de acordo com o método proposto por Cruz (2007) em que produto da tarifa de energia ( $R \$ / K w h)$ pelo montante de energia não interrompida (Kwh) fornecerão a perda de faturamento da empresa, conforme ilustra a Equação 3.1.

Perda de faturamento $=(\mathrm{R} \$ / \mathrm{Kwh})^{*}(\mathrm{Kwh})$

Em que: $(\mathrm{R} \$ / \mathrm{K} w h)$ é o produto da tarifa de energia; (Kwh) montante da energia interrompida

Para tanto, os valores referentes às quantidades de energia interrompidas, serão transformadas de Mwh para Kwh através da seguinte consideração: $1 \mathrm{Kwh}=$ 0,001 Mwh e, posteriormente, calculadas as perdas de faturamento através da Equação 3.1. 
Foram calculados os custos de interrupção entre os anos de 1998 a 2009. A nível deste trabalho serão abordadas apenas as perdas de faturamento associadas à energia não suprida, através das tarifas médias de fornecimento por classe de consumo extraídas de Bonini, (2011), DIEESE, (2007) e relatórios da ANEEL dispostos no endereço eletrônico foram consideradas para o cálculo dos custos de energia elétrica.

É importante destacar que há na literatura fórmulas que auxiliam na identificação do custo de interrupção de energia a nível nacional e regional, a exemplo do trabalho de LaCommare et al., (2004). Porém, pela indisponibilidade de dados referentes a custos para realização desta análise, optou-se por se fazer uma estimação dos mesmos, para mostrar à empresa estudada, o quanto ela perde em faturamento com os blecautes.

De posse dessas considerações, a realização da estimativa dos custos pautados às interrupções de energia elétrica indicarão qual a perda no faturamento da empresa distribuidora de energia entre 1998 e 2009, através da relação tarifa de energia $(R \$ / K w h)$ e montante de energia interrompida (Kwh). Esses valores serão indicadores de quanto o índice de Mwh, provocado pelos erros humanos influencia nos custos da empresa e gera uma perda no faturamento que pode ser bastante significativa.

\section{RESULTADOS E DISCUSSÃO}

\subsection{Análise dos Dados: Análise de Agrupamentos}

Após a adaptação do modelo com a inclusão do tipo de erro "lapso" a estrutura taxonômica adquire a seguinte configuração apresentada no Quadro 4.1.

Com a taxonomia ampliada foi aplicada a análise de agrupamentos através do software R, com o objetivo de conhecer os erros que interferem na interrupção de energia elétrica e quais eram mais evidentes. Com base nos algoritmos de agrupamentos, para os métodos hierárquicos foram aplicados o método simples, completo, das médias, do centróide, Ward e para os métodos não hierárquicos foram aplicados o método das k-médias e medóide, com o objetivo de comparar os indivíduos que possuem características mais semelhantes em relação ao processo de Berliner, modos de erro, comportamentos elementares.

Quanto ao nível de erro (ou domínio de erro) por estar diretamente relacionada ao modo de erro e seus desdobramentos este nível não foi observado. Observou-se também a variável que quantifica a energia elétrica que deixou de ser fornecida (Mwh) no erro humano e que acarretam a interrupção de energia elétrica. 
Quadro 4.1 - Taxonomia ampliada

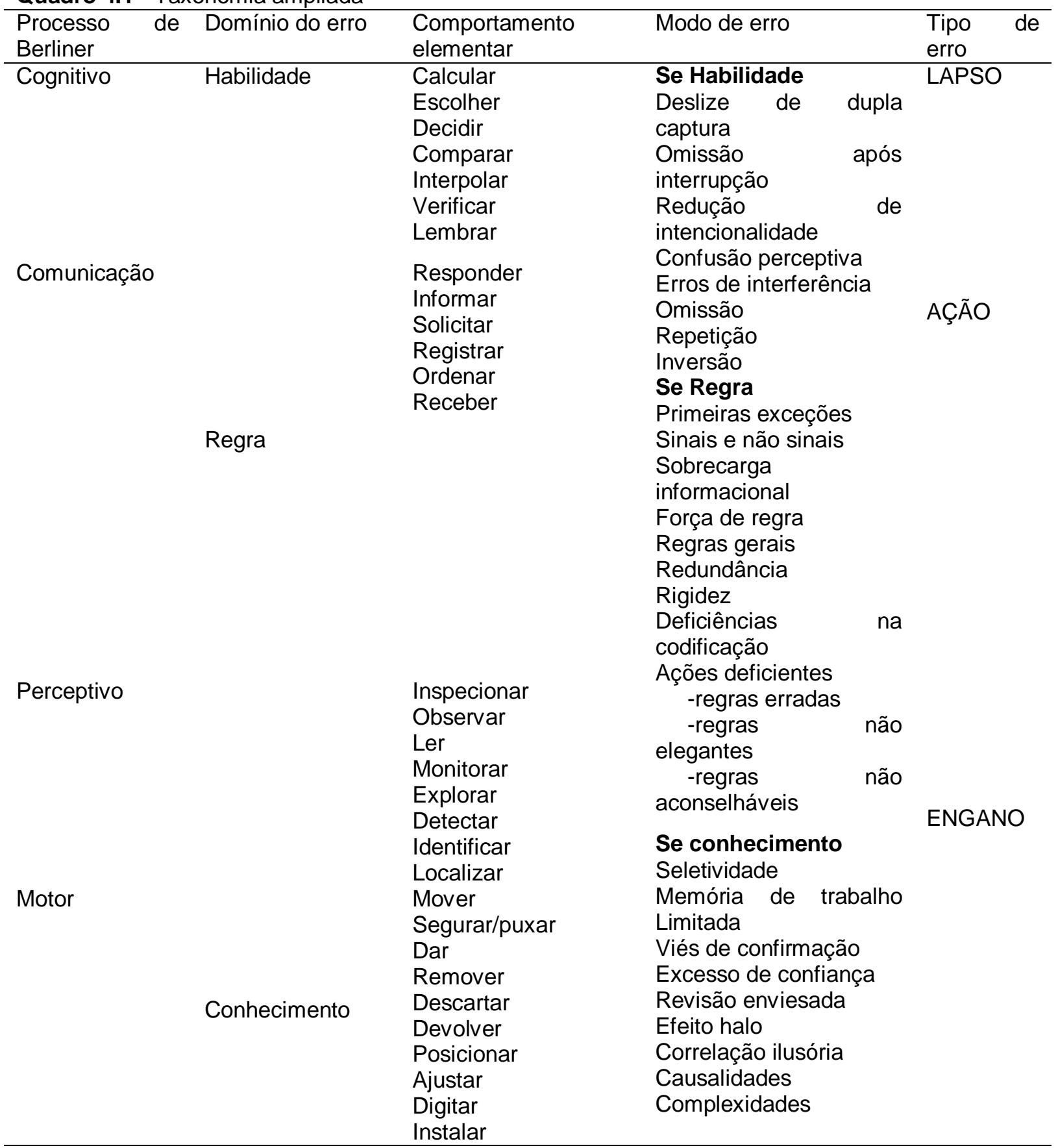

Fonte: Adaptado de Vilela (2013)

Foram realizados os agrupamentos com 2, 3, 4 e 5 grupos para os métodos hierárquicos e não hierárquicos e usada a distância euclidiana para compor a matriz de distâncias. Para todos os grupos com $\mathrm{K}=2$, 3, 4 e 5 grupos foram construídos os gráficos de silhueta em que, estes ilustraram valores negativos, o que implica em uma má alocação dos dados nos grupos, isto é, alguns elementos estão, em média, mais próximos dos elementos do grupo vizinho do que do seu próprio grupo, conforme ilustra a Figura 4.1 a seguir. 
Figura 4.1 - Gráficos de silhueta para os métodos hierárquicos com K=2
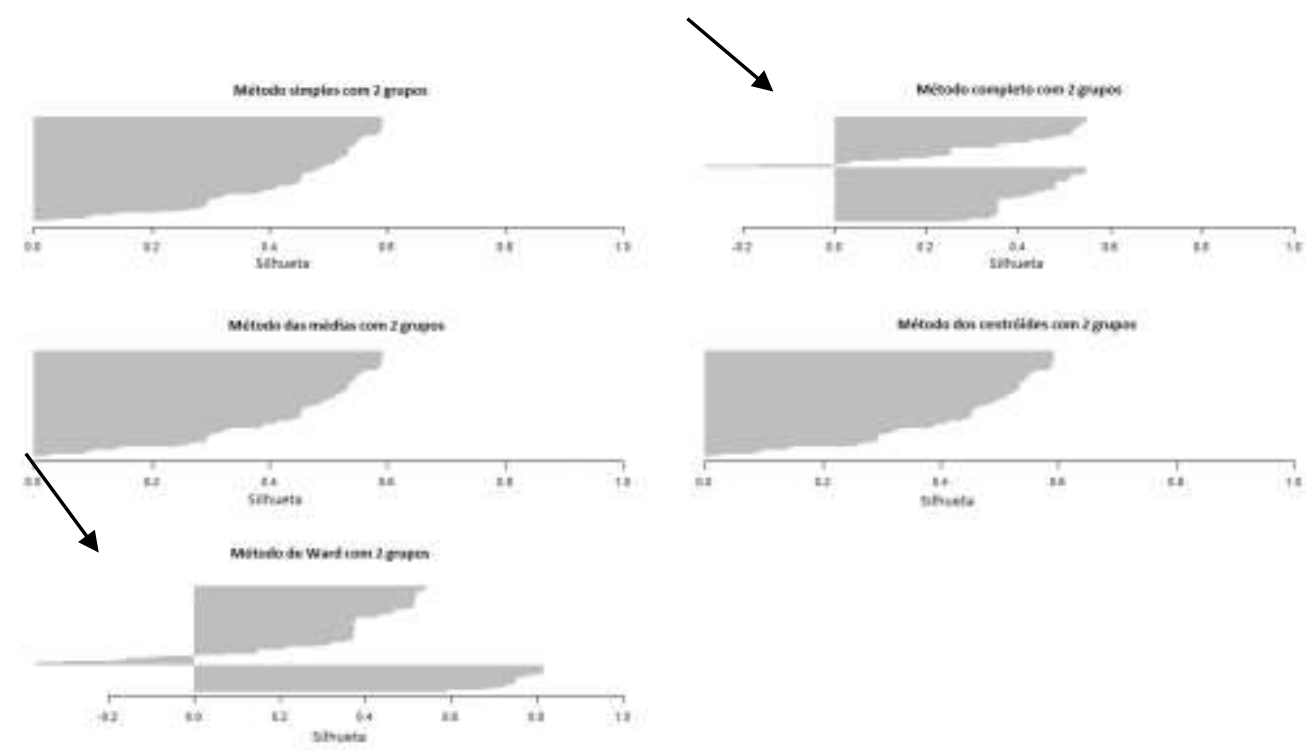

Já os métodos simples, das médias e dos centróides para $K=2$ grupos, forneceram um bom agrupamento de dados, porém, quando calculado o coeficiente cofenético, obtiveram-se os seguintes valores de correlação cofenética, conforme mostra a Tabela 4.1:

Tabela 4.1 - Valores para o Coeficiente de Correlação Cofenético

\begin{tabular}{ccc} 
& Métodos & \\
\hline Simples & Médias & Centróide \\
0,5719 & 0,8179 & 0,8218
\end{tabular}

Fonte: O Autor

Conforme observado na Tabela 4.1, o método do centróide foi o que obteve melhor desempenho de acordo com o coeficiente de correlação cofenético assim, dentre os métodos hierárquicos, ele foi escolhido.

De posse do valor coeficiente de correlação para o método do centróide, construiu-se um gráfico de perfil para mensurar a qualidade desse agrupamento com $\mathrm{K}=2$. Na Figura 4.2, observa-se $\circ$ gráfico de perfil para $\circ$ referido agrupamento encontram-se no eixo das abscissas $(x)$ e no eixo das ordenadas (y) estão as médias das variáveis de acordo com cada grupo, como mostra a Figura 4.2: 
Figura 4.2 - Gráfico de Perfil para o método do centróide com K=2

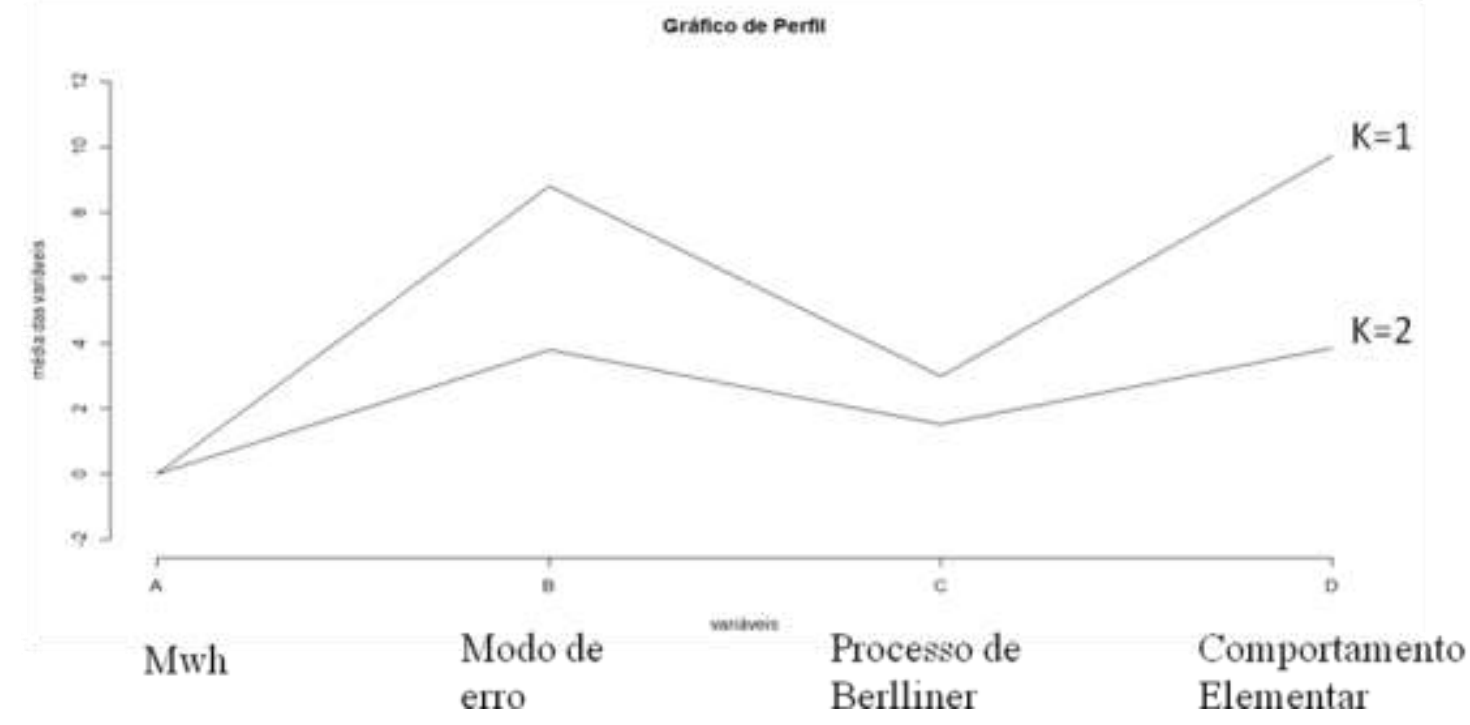

O Gráfico de Perfil ilustra que os comportamentos dos grupos de acordo com as variáveis estudadas, são semelhantes, ou seja, para a variável Mwh ambos os grupos possuem a mesma média, próximos a zero. Para o processo de Berliner, a diferença entre as médias foi pequena. Já para as demais variáveis modo de erro e comportamento elementar a diferença entre as médias dos grupos é considerável, todavia, o comportamento de crescimento e decrescimento das médias das variáveis entre os grupos é o mesmo. Isso faz com que esses grupos não estejam tão distintos assim e portanto, $\mathrm{K}=2$ não seja significativo para a análise.

Como os métodos hierárquicos não apresentaram resultados satisfatórios, deu-se início ao procedimento dos métodos não hierárquicos. Para saber com quantos grupos a análise seria iniciada, através dos métodos não hierárquicos, usou-se o índice $G$ mencionado na Equação 2.1. Assim, observando a Figura 4.3, vemos que $G$ estabiliza, ao redor do número 5 , porém não há muita vantagem em passar de quatro para cinco grupos, pois pode haver repetição das características em um quinto grupo, logo uma solução com quatro grupos é ideal. 
Figura 4.3 - Valores para o índice G

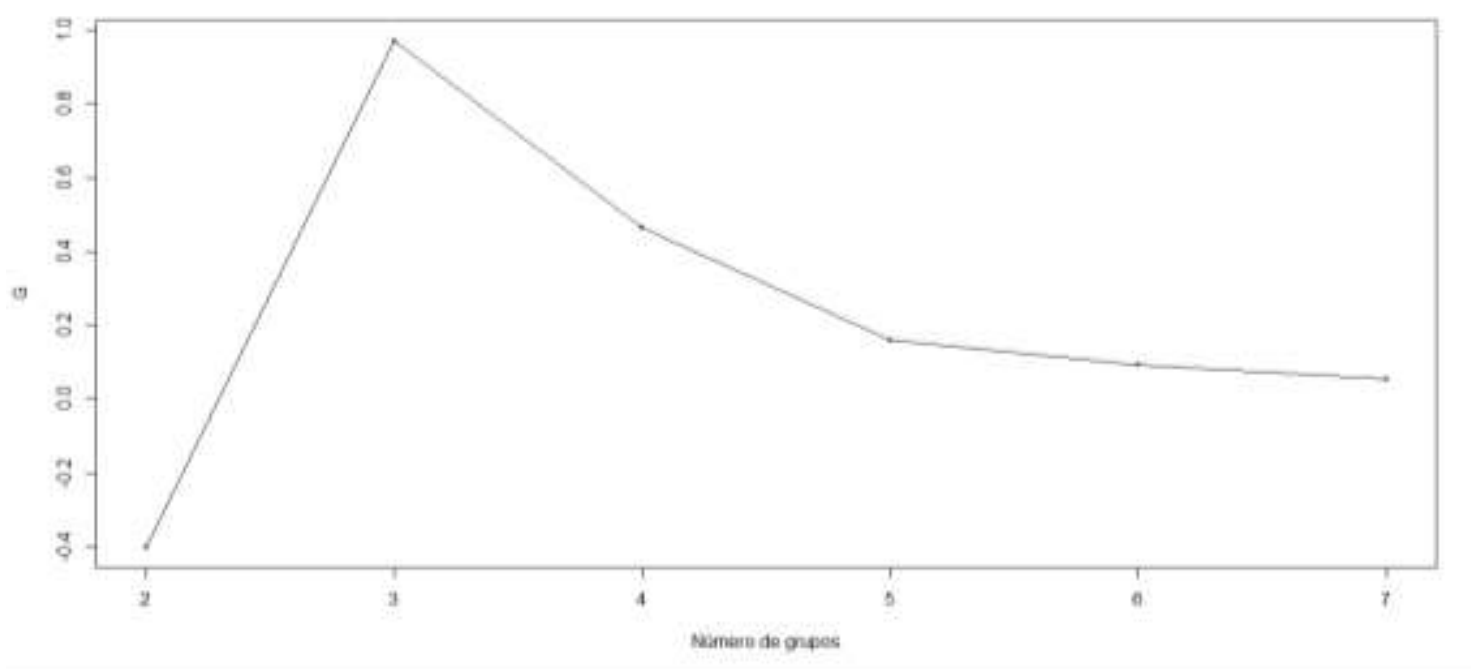

Ainda que o índice G da Figura 4.3 apresente que o melhor agrupamento é com $K=4$, foi realizado o procedimento das $k$-médias para $K=2,3$ e 5 , os quais apresentaram valores negativos no gráfico de silhueta. Nesse sentido a utilização do agrupamento $\mathrm{K}=4$ foi satisfatório, pois nenhum valor da silhueta foi negativo, fortalecendo o anteriormente relatado com o índice $\mathrm{G}$ e explicitado na Figura 4.4.

Figura 4.4 - Gráficos de silhueta para o método não hierárquico das K-médias, com $\mathrm{K}=2$, $\mathrm{K}=3$, $\mathrm{K}=4$ e $\mathrm{K}=5$
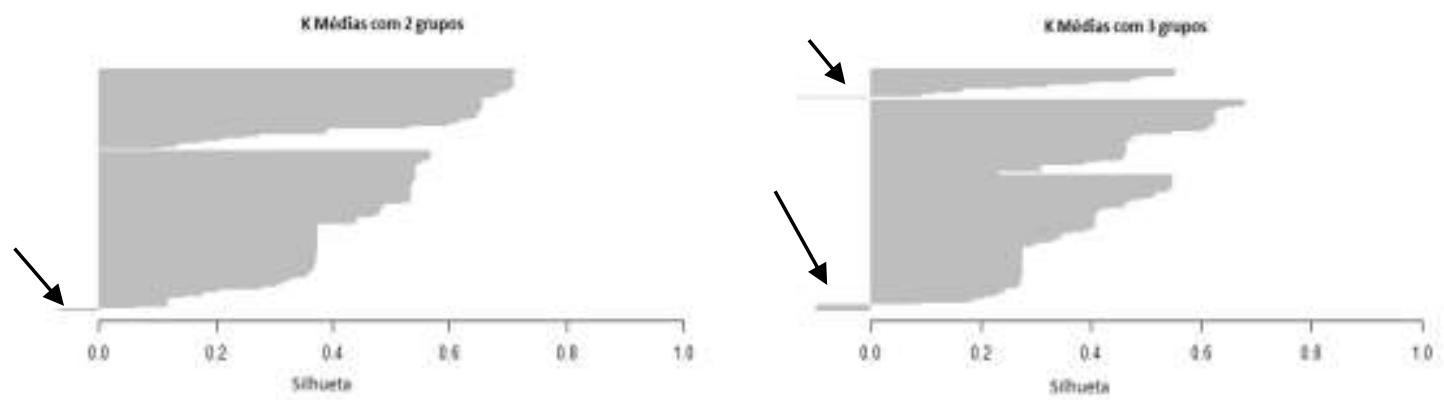

KMediasemen 4 grugos

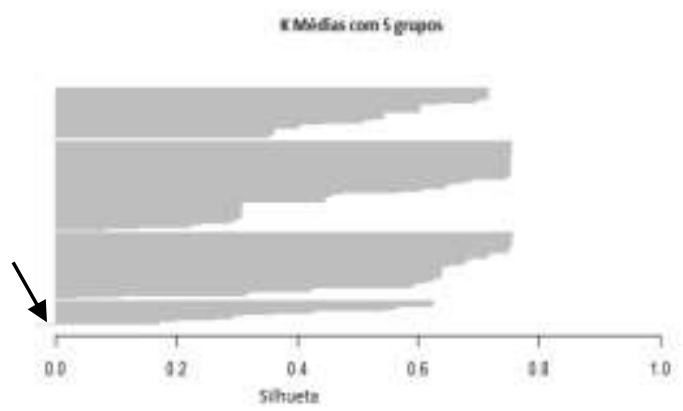

Os agrupamentos realizados a partir do método k-medóides com 2, 3, 4 e 5 grupos, não tiveram uma boa representação no gráfico da silhueta, apresentando nestes, valores negativos, conforme ilustra o Figura 4.5. 
Figura 4.5 - Gráficos de silhueta para o método não hierárquico k-medóide
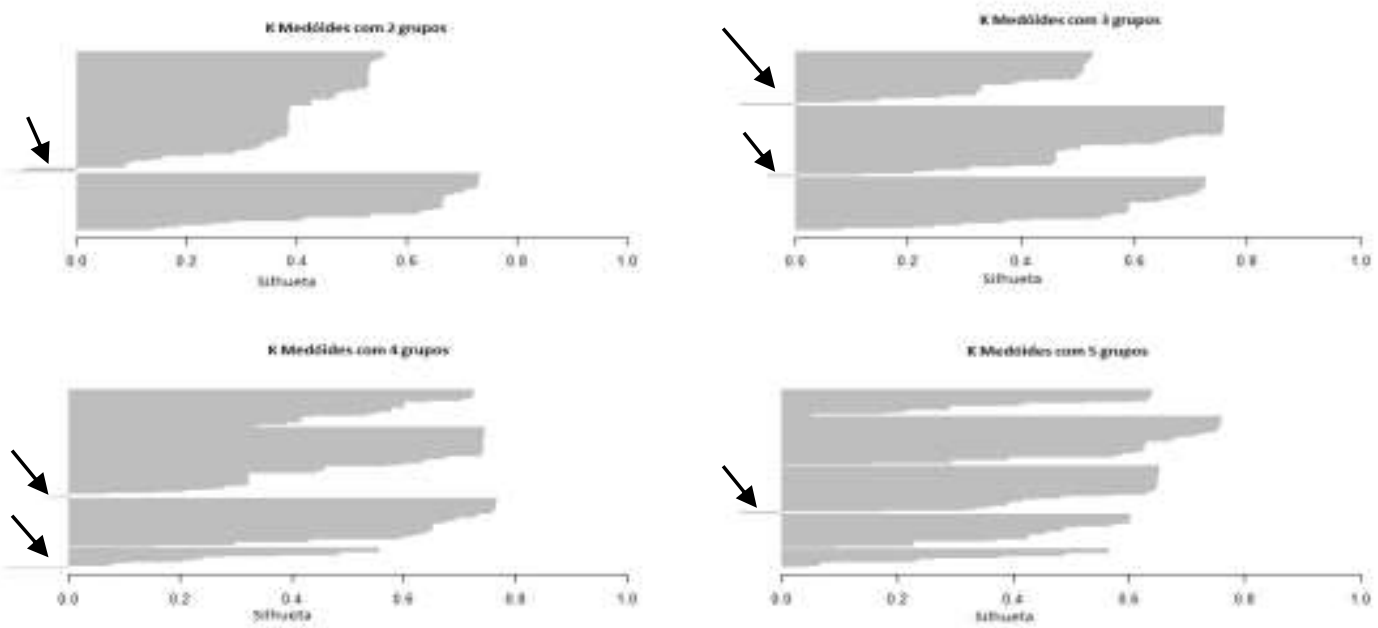

Como o método do medóide não apresentou bom desempenho, escolheu-se o método das k-médias para análise dos agrupamentos, usando $\mathrm{K}=4$, para os fatores que podem ser os possíveis causadores de interrupção de energia elétrica.

As análises dos quatro grupos foram realizadas baseadas nas semelhanças entre os elementos de cada grupo e as diferenças entre eles. Observou-se que os grupos são responsáveis por uma determinada quantidade de energia que deixou de ser fornecida, sendo influenciados pelos comportamentos elementares dos indivíduos que por sua vez, tem um conjunto de elementos que são influenciadores do erro. A Tabela 4.2 apresenta um resumo das variáveis observadas em relação à porcentagem da carga total interrompida.

De acordo com o exposto, tem-se a porcentagem de carga total interrompida por cada grupo, em relação a cada categoria, como por exemplo: o grupo 2 teve um total de 1030,46 Mwh de carga interrompida, do qual 801,07 Mwh foi devido ao processo motor, o que corresponde a $801,07 / 1030,46=77,74 \%$ do total desse grupo.

Percebe-se que há uma grande repetição de algumas das características da taxonomia ampliada, nos agrupamentos com $\mathrm{K}=2$, 3 e 4 grupos, e que causam grandes quantidades de interrupção de energia (Mwh), tendo o processo cognitivo o maior índice de ocorrências entre os três grupos, com $94,73 \%$, o modo de erro "confusão perceptiva" que totalizou a maior quantidade de energia interrompida 2064,94 Mwh, o comportamento "comparar" ocasionou a parada 1691,91 Mwh de energia e o tipo de erro "ação" foi o de maior incidência dos erros humanos com $77,47 \%$ de Mwh interrompido. 
Tabela 4.2 - Resumo das variáveis observadas em relação à porcentagem da carga total interrompida

\begin{tabular}{|c|c|c|c|c|c|}
\hline & & $\begin{array}{l}1 \text { elemento; } \\
1402,24\end{array}$ & $\begin{array}{l}38 \text { elementos; } \\
1030,46 \mathrm{Mwh}\end{array}$ & $\begin{array}{l}\text { 51 elementos; } \\
\text { 2133,25 Mwh }\end{array}$ & $\begin{array}{l}41 \text { elementos; } \\
2341,69 \mathrm{Mwh}\end{array}$ \\
\hline \multirow{2}{*}{$\begin{array}{c}\text { Variáveis } \\
\text { Estudadas }\end{array}$} & \multicolumn{2}{|c|}{ Categorias Grupo } & $\sqrt{\text { Grupo }}$ & Grupo & $\sqrt{\text { Grupo } 4}$ \\
\hline & Cognitivo & \multicolumn{4}{|c|}{ Porcentagem da carga total interrompida (\%) } \\
\hline Berlliner & $\begin{array}{c}\text { Motor } \\
\text { Perceptivo }\end{array}$ & 100 & 77,71 & $\begin{array}{l}27,32 \\
69,92\end{array}$ & 0,40 \\
\hline \multirow{8}{*}{$\begin{array}{l}\text { Comportamento } \\
\text { Elementar }\end{array}$} & $\begin{array}{l}\text { Sinais e não } \\
\text { sinais }\end{array}$ & 100 & & & \\
\hline & Remover & & 71,77 & & \\
\hline & Relembrar & & 22,16 & & \\
\hline & Identificar & & & 69,92 & \\
\hline & Mover & & & 27,71 & \\
\hline & Comparar & & & & 72,24 \\
\hline & Decidir & & & & 21,61 \\
\hline & Inspecionar & & & & 4,76 \\
\hline \multirow{3}{*}{ Tipos de erro } & Ação & 100 & 77,47 & & \\
\hline & Lapso & & & & \\
\hline & Engano & & & 76,80 & 76,67 \\
\hline \multirow{6}{*}{ Modos de Erro } & $\begin{array}{l}\text { Confusão } \\
\text { perceptual }\end{array}$ & & & 96,80 & \\
\hline & $\begin{array}{l}\text { Revisão } \\
\text { tendenciosa }\end{array}$ & & 4,01 & & 64,86 \\
\hline & Força & 100 & & 1,66 & \\
\hline & regra & & & & \\
\hline & Ações & & & & 8,35 \\
\hline & $\begin{array}{l}\text { deficientes } \\
\text { Deficiências } \\
\text { na } \\
\text { codificação }\end{array}$ & & & & 14,88 \\
\hline
\end{tabular}

Fonte: O Autor

Considera-se que os erros humanos advêm inicialmente de uma das categorias dispostas no processo de Berliner e que resultam em um encadeamento dos outros quatro domínios do erro (tipo de erro, nível de erro, comportamento elementar e modo de erro). Quando ocorre do indivíduo ter o processo cognitivo como o elemento ocasionador dos erros, entende-se que no momento de decisão sobre qual regra usar para uma determinada atividade escolheu inconscientemente, a regra mais forte em detrimento da mais fraca, quando não necessariamente a regra mais forte seria a aplicável a determinada situação. Decorrente da cognição, o 
comportamento elementar "comparar", que está relacionado à identificação de semelhanças ou diferenças entre dois ou mais objetos, foi a de maior ocorrência, devido à dificuldade em tomar a decisão correta quando na escolha entre dois ou mais elementos.

Nesse sentido, fica claro que o trabalhador não possui conhecimentos específicos sobre a(s) atividade(s) que está (ão) desempenhando e supõem que uma determinada regra, se enquadra em todas as outras que the são semelhantes. Neste cenário, a confusão perceptiva se faz presente quando considera-se que na rotina de trabalho, os indivíduos realizam suas atividades sem prestar atenção no que estão fazendo como por exemplo: acionar um dispositivo que está em uma posição próxima a que está acostumado a operar ou um dispositivo similar, que ocasionará o disparo de uma regra que não se enquadra ideal.

Nesse ínterim Begosso (2005) afirma que um modelo que descreve os tipos de erro compatíveis com o modelo de processamento humano de Rasmussen, Reason e Berliner, fornece um modelo de erros mais amplo com uma maior discriminação dos erros.

\subsection{Estimação dos custos de energia elétrica.}

A perda no faturamento de energia elétrica está ilustrado na Tabela $4.3 \mathrm{em}$ que, o produto tarifa de energia $(\mathrm{R} \$ / \mathrm{K} w \mathrm{w})$ por energia interrompida (Kwh) fornecerá o custo da interrupção de energia ao longo dos anos estudados (de 1998 a 2009), bem como o total da perda de faturamento ao logo deste período devido aos blecautes ocorridos.

Tabela 4.3 - Perdas de faturamento associadas à interrupção de energia, através das tarifas médias de fornecimento

\begin{tabular}{cccc}
\hline ANO & $\begin{array}{c}\text { Tarifa de energia } \\
(\mathbf{R} \mathbf{\$} / \mathbf{K w h})\end{array}$ & $\begin{array}{c}\text { Energia interrompida } \\
\mathbf{( K w h )}\end{array}$ & Perda no faturamento \\
\hline $\mathbf{1 9 9 8}$ & 86,57 & 145090 & $12.560 .441,30$ \\
$\mathbf{1 9 9 9}$ & 95,86 & 166020 & $15.914 .677,20$ \\
$\mathbf{2 0 0 0}$ & 108,50 & 862800 & $93.613 .800,00$ \\
$\mathbf{2 0 0 1}$ & 122,88 & 256160 & $31.476 .940,80$ \\
$\mathbf{2 0 0 2}$ & 143,05 & 1711970 & $244.897 .308,50$ \\
$\mathbf{2 0 0 3}$ & 183,39 & 522710 & $95.859 .786,90$ \\
$\mathbf{2 0 0 4}$ & 214,71 & 539810 & $115.902 .605,10$ \\
$\mathbf{2 0 0 5}$ & 244,09 & 969350 & $236.608 .641,50$ \\
$\mathbf{2 0 0 6}$ & 258,29 & 830390 & $214.481 .433,10$ \\
$\mathbf{2 0 0 7}$ & 260,49 & 265840 & $69.248 .661,60$ \\
$\mathbf{2 0 0 8}$ & 248,45 & 46740 & $11.612 .553,00$ \\
$\mathbf{2 0 0 9}$ & 261,00 & 399230 & $104.199 .030,00$ \\
& & & $1.246 .375 .879,00$ \\
\end{tabular}

Fonte: O Autor, a partir de dados obtidos da ANEEL

De acordo com o exposto, tem-se que ao longo de 11 anos de interrupção de energia elétrica devido a erros humanos, a empresa estudada teve uma perda no 
faturamento no âmbito de $R \$ 1.246 .375 .879,00$ (um bilhão, duzentos e quarenta e seis milhões, trezentos e setenta e cinco mil, oitocentos e setenta e nove reais).

Os custos calculados são apenas estimativas e podem estar sub ou super mensurados devido a coleta de dados, as interpretação dos registros de erro humano, da realização da análise de agrupamentos, mas que de alguma forma refletem o quanto a empresa estudada tem de prejuízo por causa dos erros humanos. Os erros humanos referentes aos anos de 2010, 2011, 2012 e 2013 não foram calculados devido a indisponibilidade dos dados referentes a esses anos.

\section{CONSIDERAÇÕES FINAIS}

A contribuição para este trabalho está fundamentada na inclusão do tipo de erro "lapso" ao modelo de identificação dos erros humanos onde seu principal objetivo foi o de verificar a incidência desses erros devido à falta de atenção e/ou falha de memória e que são causadores de interrupção de energia elétrica, juntamente com uma estimativa dos custos associados a esses erros.

Através da realização da análise de agrupamento, o grupo 4 foi o que apresentou uma maior quantidade de energia interrompida com 2341,69 Mwh e, dentro os elementos constituintes do processo de Berliner, o processo cognitivo foi o maior motivador das interrupções com $94,73 \%$ de incidência, assim como o comportamento elementar de "comparar" com 1691,61 Mwh de energia interrompida. Em contrapartida, a maior interrupção por parte do tipo de erro está no grupo 2, com o tipo de erro ação com 77,47\% das ocorrências ou 798,28 Mwh.

Devido às altas porcentagens de erros humanos para os 131 casos de erros humanos estudados, a estimativa dos custos decorrentes das interrupções de energia apresentaram o valor de $R \$ 1.246 .375 .879,00$ (um bilhão, duzentos $e$ quarenta e seis milhões, trezentos e setenta e cinco mil, oitocentos e setenta e nove reais), referentes aos anos de 1998 a 2009, o que reforça ainda mais a idéia da relação de erros humanos com o aspecto econômico. Diante dessa assertiva, os erros humanos devem ser observados à luz dos modos de erro que ocasionam interrupções de energia elétrica e estes devem ser minimizados de modo que a perda de faturamento seja reduzida, quando não evitada.

Diante destas análises, conclui-se que os erros humanos ocorrem em sua maneira quando há a violação de alguma regra que gera uma decisão equivocada e que, por conseqüência acarreta as interrupções de energia. Percebe-se, no entanto, que os erros apresentam limitações no que concerne ao comportamento humano, dado a inviabilidade de se conhecer/saber o que se passa com cada indivíduo em seu âmbito tanto pessoal quanto profissional, e que interferem na execução de suas atividades.

\section{REFERÊNCIAS}

ANDERBERG, M. R. Cluster analysis for applications. London: Academic Press, 1973. 
ALBUQUERQUE, M. A. Estabilidade em análise de agrupamento (cluster analysis). Dissertação apresentada ao Programa de Pós Graduação da Universidade Federal Rural de Pernambuco. Recife, 2005.

BARROSO, L. P.; ARTES, R. Análise multivariada: Minicurso do 10 Simpósio de estatística Aplicada A experimentação Agronômica - RBRAS, 48 reunião Anual da região Brasileira da Sociedade Internacional de biometria - SEAGRO. Lavras: UFLA, 2003.

BEGOSSO, L. C. S. PERERE. Uma ferramenta apoiada por arquiteturas cognitivas para o estudo da confiabilidade humana. Engenharia Elétrica. São Paulo, Escola Politécnica da Universidade de São Paulo, 2005.

BERLINER, D. C., D. Angell et al. Behaviors, measures and instruments for performance evaluation in simulated environments. Symposium and Workshop on the Quantification of Human Performance. Albuquerque - New Mexico, p. 277-296 , 1964.

BONINI, M. R. Tarifas de energia elétrica: evolução nos últimos anos e perspectivas. Boletim de economia. Outubro de 2011.

BRITO, W. M.; SEMAAN, G. S.; BRITO, J. A. M. Um Algoritmo Genético para o Problema dos K-Médoides. 10th Brazilian Congress on Computational Intelligence (CBIC'2011), November 8 to 11, 2011, Fortaleza, Ceará Brazil.

BUSSAB, W. DE O; MIAZAKI, E. S; ANDRADE, D. Introdução à análise de agrupamentos. São Paulo: Associação Brasileira de Estatística, 1990.

CALIXTO, E. Notas de aula da disciplina de gerenciamento e análise de risco. Curso de Especialização de Engenharia de Segurança do Trabalho. PUC,Rio de Janeiro, 2011.

CARVALHO, P.V. R.; OLIVEIRA M. V.; SANTOS I. L.; VIDAL, M. C. R. A Modelagem Cognitiva e a Confiabilidade Humana em Organizações que Lidam com Tecnologias de Risco. ENCONTRO NACIONAL DE ENGENHARIA DE PRODUÇÃO CURITIBA, 22, 2002,. Anais... Curitiba, PR, 2002.

CORREA, C. R. P.; JUNIOR, M. M. C. Análise e classificação dos fatores humanos nos acidentes industriais. Produção, v. 17, n. 1, p. 186-198, Jan./Abr. 2007. https://doi.org/10.1590/S0103-65132007000100013

CRUZ, M. P. Metodologia para avaliação dos impactos econômicos associados a problemas de qualidade de energia. Dissertação (Mestrado) - Universidade Federal de Santa Catarina. Florianópolis, 2007.

DIEESE. Departamento Intersindical de Estatística e Estudos Sócio-Econômicos. As tarifas de energia elétrica no Brasil: sistemática de correção e evolução dos valores. Nota técnica 58. Dezembro, 2007.

DONI, M. V. Análise de cluster: métodos hierárquicos e de particionamento. Monografia apresentada a Universidade Presbiteriana Mackenzie. São Paulo, 2004.

DROGUETT, E.L. MENÊZES R.C.L. Análise da confiabilidade humana via redes Bayesianas: uma aplicação à manutenção de linhas de transmissão. Produção, v. 17, n. 1, p. 162-185, Jan./Abr. 2007. https://doi.org/10.1590/S0103-65132007000100012 
FILHO, A. C. G.; VANZIN, T.; FORCELLINI, F. A. Erros humanos: considerações sob um ponto de vista cognitivo aplicado a processos criativos de negócios. Ciências \& Cognição, v. 14, n. 1 , p. 219-232, 2009.

FREI, F. Introdução à análise de agrupamentos. São Paulo: Editora UNESP, 2006.

FILGUEIRAS, L.V.L. Método para analise e projeto de interfaces homem - computador visando confiabilidade humana. 1996. 259 f. Tese (Doutora em Engenharia) - Departamento de Engenharia de Computação e Sistemas Digitais, Escola Politécnica da Universidade de São Paulo.

LACOMMARE, K. H; Eto, J. Understanding the cost of power interruptions to U.S. Electricity Consumers. Lawrence Berkeley National Laboratory. 2004. https://doi.org/10.2172/834270

LINDEN, R. Técnicas de Agrupamento. Revista de Sistemas de Informação da FSMA, n. 4, p. 18-36, 2009.

MATOS, R. A. Comparação de metodologias de análise de agrupamentos na presença de variáveis categóricas e contínuas. Dissertação (Mestrado) -Programa de PósGraduação em Estatística da Universidade Federal de Minas Gerais. Belo Horizonte, 2007.

MARTINS, R. M. Utilização de técnicas de análise de agrupamento do risco de geada no estado do paraná para a cultura do milho safrinha. Tese (Doutorado) - Universidade Estadual Paulista. Botucatu, 2008.

MENÊZES, R. C. S. Uma metodologia para avaliação da confiabilidade humana em atividades de substituição de cadeias de isoladores em linhas de transmissão. Dissertação (Mestrado) - Universidade Federal de Pernambuco, Recife, 2005.

MEYER, A. S. Comparação de Coeficientes de similaridade usados em análise de agrupamentos com dados de marcadores moleculares dominantes. Dissertação (Mestrado) - Universidade de São Paulo. Piracicaba, 2002. https://doi.org/10.11606/d.11.2002.tde-24072002-165250

RASMUSSEN, J. What can be learned from human error reports? In Changes in Working Life. Wiley. London, 1980.

REASON, J. Human Error. Cambridge, Cambridge University Press. 1990. https://doi.org/10.1017/CBO9781139062367

RIBEIRO, A. C. O. Quantificação do impacto de fatores humanos e organizacionais em probabilidade de falha humana usadas em análise probabilística humana. Tese (Doutorado). Rio de Janeiro, 2012.

RIGHI, A.W.; GONÇALVES, L. L. G.; WACHS, P.; JUNIOR, G. C. C.; SAURIN, T. A. Erros humanos em acidentes de trabalho: um estudo de caso no setor elétrico. ENCONTRO NACIONAL DE ENGENHARIA DE PRODUÇÃO,30., 2010. Maturidade e desafios da Engenharia de Produção: competitividade das empresas, condições de trabalho, meio ambiente. São Carlos, SP. Anais...., Brasil, 12 a15 de outubro de 2010.

SANDERS, M.S.; McCormick, E.J. Human factors in engineering and design. 7 ed. New Youk: Mc Graw-Hill, 1993. 
SANTOS, M. A. Ferramenta de análise de riscos em projetos de capital considerando conceitos de confiabilidade humana e engenharia de resiliência. Dissertação

(Mestrado) - Programa de Pós Graduação da Universidade Federal do Rio de Janeiro. Rio de Janeiro, 2012.

SHIGA, A. A., PIANTINI, A., PEGOLLO, C. A. G. Considerações sobre os custos decorrentes de descargas atmosféricas em sistemas de distribuição de energia.

SEMINÁRIO NACIONAL DE DISTRIBUIÇÃO DE ENERGIA ELÉTRICA, 17., 2006. Anais... Belo Horizonte, 2006.

SICILIANO, R. S.; FERRAZ, F. T. Análise da Confiabilidade Humana Requer Maturidade Organizacional na Escolha da Ferramenta. Encontro Nacional de Engenharia de Produção. Maturidade e desafios da Engenharia de Produção: competitividade das empresas, condições de trabalho, meio ambiente, 30., 2010. Anais... São Carlos, SP, Brasil, 12 a15 de outubro de 2010.

SWAIN, A D; GUTTMANN, H. E. Handbook of human reliability analysis with emphasis on nuclear power plant applications. US Nuclear Regulatory Commission. Washington, 1983.

VICINI, L.; SOUZA, A. M. Análise multivariada da teoria à prática. Monografia apresentada ao curso de especialização da Universidade Federal de Santa. Maria Santa Maria, RS, 2005.

VILELA, R. T. New Taxonomy and model of error sequence process for human error assessement in hydroelectric power systems. Tese submetida ao programa de Pós Graduação da Universidade Federal de Pernambuco UFPE. RECIFE, 2013.

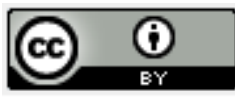

Artigo recebido em 12/05/2014 e aceito para publicação em 01/11/2016 DOI: http://dx.doi.org/ 10.14488/1676-1901.v16i4.1795 